\title{
An inexpensive, efficient cage for small birds
}

\section{RONALD KALINOSKI and JOHN P. GLUCK \\ University of New Mexico, Albuquerque, New Mexico 87104}

A simple cage system is described which is particularly applicable for housing small birds such as sparrows and finches that have been trapped in the wild. The cages are constructed of inexpensive, readily available materials.

Even a cursory perusal of the comparative literature reveals the existence of large species sample gaps (Beach, 1950; Lockard, 1971). Certainly, one of the most obvious of these omissions is the restricted samples of bird populations present in the literature. Commercially available laboratory housing is primarily designed for relatively large birds such as pigeons and quail. The researcher wishing to use small birds such as finches or sparrows is faced with the decision of either modifying what is commercially available or purchasing pet bird caging. Either alternative requires a significant financial outlay and may not be appropriate to the species, since vertical space is a more important dimension than is horizontal space to most birds. We have recently initiated a series of behavioral studies with small passerine birds trapped in the wild which has resulted in the design of an inexpensive, efficient environment for the housing of individuals and small groups of up to three or four birds. The cage, illustrated in Fig. 1, is $26 \mathrm{in}$. in diam and $20 \mathrm{in}$. high and is composed of six nylon panels placed over a spring steel frame attached to a hexagonal reinforcing bar base. The cage contains a natural branch perch suspended from the zenith and is placed over waste-absorbent paper. A vertically aligned and centered nylon zipper sewn into one of the panels serves as the access door.

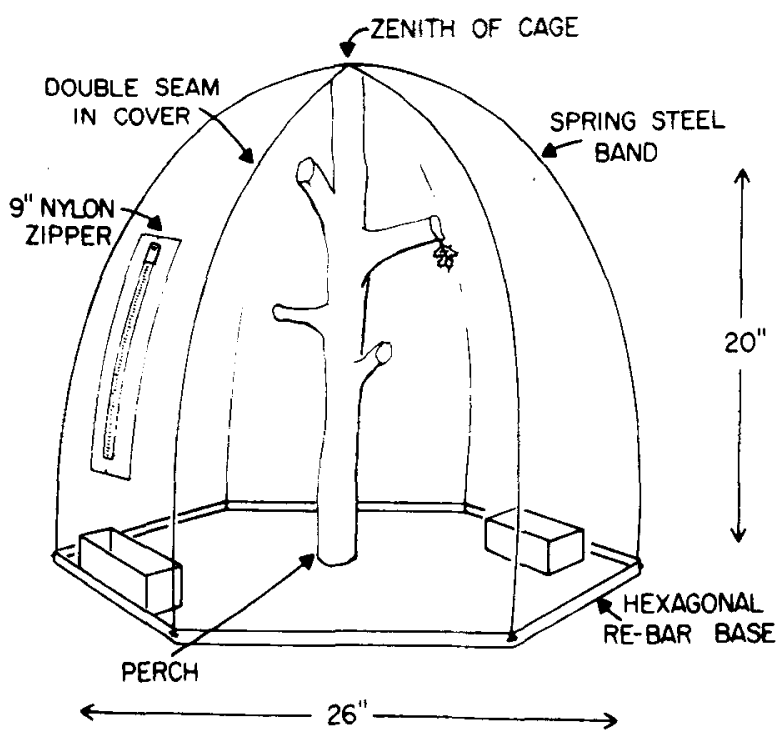

Fig. 1. Housing cage for small birds.

\section{CONSTRUCTION}

A list of materials and approximate costs is provided in Table 1. The base of the cage is constructed by bending an 80 -in. length of $1 / 4$-in. reinforcing bar rod into six equal 13 -in. sides at $60 \mathrm{deg}$ to one another. The ends of the base may be joined by welding, tape, or metal glue. Three spring steel strips, $1 / 4$ in. wide $\times 1 / 16$ in. thick $x 56$ in. in length and cut from a pair of inexpensive pants stretchers, are flexed and attached by crimping to opposite corners of the cage base. A short piece of soft electrical wire is wrapped around the intersection of the three bands at the zenith of the cage to prevent frame slippage. Once the frame is completed, a nylon side panel pattern is eonstructed by tracing along two adjacent bands onto a sheet of newsprint. Using this pattern, six panels are cut from a sheet of stretchable transparent nylon material which can be found at any fabric store. A border of approximately $1 / 2$ in. is cut around the pattern for a seam allowance. A 9-in. zipper, aligned vertically and centered into one of the side panels, is then installed. The panels are then machine sewn together, using a doubly reinforced flat-felled seam. In order to get the cover to fit securely around the base of the cage, a $1 / 4$-in. binding elastic is sewn completely around the base of the cover. When installing the elastic band, it must be pulled taut so that when it is relaxed there is a tendency for the material to pucker. The best perches to use with this cage are natural wood branches that possess several horizontal side branches. The branches are cut to fit snugly from the floor of the cage to the zenith. Excess length of the side branches must be trimmed so that they do not touch the cover. Final assembly involves simply pulling the cover over the frame so that the seams are aligned on top of the steel bands. The elastic sewn into the margin of the cover bottom should then be pulled over the base. With the addition of food, water, and grit dishes, the cage is ready for occupancy. If many cages are to be

Table 1

Materials and Approximate Costs for Passerine Housing

1 Pants stretchers, large size. Each pair of stretchers can be taken apart to give four spring steel bands about $56 \times 1 / 4 \times 1 / 16$ in. thick. Cost: \$1.00/pair.

2 Re-bar, $1 / 4$ in. smooth. Can be bought in $20-\mathrm{ft}$ lengths at any steel company. Cost: $\$ .90 / 20 \mathrm{ft}$.

3 Nylon material, transparent and stretchable. Each cage requires about 1 yd $\times 60-70 \mathrm{in}$. of material and can be purchased at a fabric store. Cost: $\$ 2.00 / \mathrm{yd}$.

4 Nylon zipper, 9-10 in. long. Obtainable at fabric store. Cost: $\$ .19$.

5 Elastic, oval. $1 / 8$ in. wide. Also can be obtained at fabric store. Four yards is good for two cages. Cost: $\$ .49 / 4 y d$.

6 Soft. flexible wire, about 8 in. per cage. 
used. simple shelves can be constructed to accommodate them efficiently.

It should be noted that the soft flexible nylon cover does not wear on the feathers of the birds as do typical wire mesh cages. This feature is quite important if the natural mobility of the birds is to be maintained. Further. when birds get excited. they cannot injure themselves by flying into the sides of the cage. The nylon cover does not snag, so the birds do not get their claws caught in the cover. The zipper-type door opening reduces considerably the chances of birds escaping while being removed or when food and water is changed. The zipper door also permits easy access to all parts of the cage, facilitating capture of birds inside the cage. Most birds are notoriously messy when kept in standard laboratory cages. These cages, however, are tapered toward the top. thus reducing significantly the accumulation of droppings on the sides. The central location of the perch, upon which the birds will roost, further contributes to this end. When necessary, the cover can be easily removed, washed, and disinfected. With regard to storage, the cages can be dismantled easily and require very little storage space. The final advantage of this cage is cost. Depending upon the availability of materials, our construction costs have been as low as $\$ .45$ to a high of $\$ 3.50$ per cage. The cages requires approximately $2-3 \mathrm{~h}$ or less to construct.

\section{REFERENCES}

Beach, F. The snark was a boojum. American Psychologist, $1950,5,115-124$.

Lockard, $R$. B. Reflections on the fall of comparative psychology: Is there a message for all of us? American Psychologist. 1971, 26, 168-179.

(Received for publication February 15. 1973; accepted February 24, 1973.) 